\title{
Article
}

\section{Research on Measurement Method of Yak Body Size and Weight Based on Convolutional Neural Network and Binocular Vision}

\author{
Wenzhi Wang ${ }^{1,+}{ }^{,}$Yuan Zhang ${ }^{1,+, \neq}$, Jie He ${ }^{1}$, Zhanqi Chen ${ }^{1}$, Dan $\mathrm{Li}^{1}{ }^{1}$, Chong $\mathrm{Ma}^{1}$, Rende Song ${ }^{2}$, Yang Ba ${ }^{2}$, Qiucuo \\ Baima $^{2}$, XiaoQin $\mathrm{Li}^{2}$ \\ 1 Department of Computer Technology and Applications, Qinghai University, Ningda Road, 810016, Xining, \\ China. \\ 2 Center for Animal Disease Control and Prevention in Yushu State, 815000, Yushu, China. \\ + WenZhi Wang and YuAn Zhang have contributed equally to this work \\ $\ddagger$ Correspondence address: 2011990029@qhu.edu.cn
}

\begin{abstract}
In order to solve the labor-intensive and time-consuming problem in the process of measuring yak body size and weight in yak breeding industry in Qinghai Province, a non-contact method for measuring yak body size and weight was proposed in this experiment, and key technologies based on semantic segmentation, binocular ranging and neural network algorithm were studied to boost the development of yak breeding industry in Qinghai Province. Main conclusions: (1) Study yak foreground image extraction, and implement yak foreground image extraction model based on U-net algorithm; select 2263 yak images for experiment, and verify that the accuracy of the model in yak image extraction is over $97 \%$. (2) Develop an algorithm for estimating yak body size based on binocular vision, and use the extraction algorithm of yak body size related measurement points combined with depth image to estimate yak body size. The final test shows that the average estimation error of body height and body oblique length is $2.6 \%$, and the average estimation error of chest depth is $5.94 \%$. (3) Study the yak weight prediction model; select the body height, body oblique length and chest depth obtained by binocular vision to estimate the yak weight; use two algorithms to establish the yak weight prediction model, and verify that the average estimation error of the model for yak weight is $10.78 \%$ and $13.01 \%$ respectively.
\end{abstract}

Keywords: yak,semantic segmentation,binocular vision,body size,weight stimation

\section{Introduction}

Pastoral areas in Qinghai Province account for $96 \%$ of the total area of the province and the yak in the province account for about $34 \%$ of yak population in the world, among which Yushu Prefecture and Guoluo Prefecture have the largest number of yaks[1]. As a unique cattle breed in the alpine region of Qinghai-Tibet Plateau, Yak mainly grow in the region of Qinghai-Tibet Plateau in China with an altitude of over 3,000 meters and an average temperature below zero. It is the main livestock and economic source of herdsmen in the plateau region [2]. Qinghai Province has the largest number of yaks in the world. After long-term reproduction and growing in natural closed environment in various regions of the province, species resources such as Qinghai plateau yak, Huanhu yak and Xueduo yak with high consistency in body shape and economic characters and stable genetic performance have been formed [3]. In the protection and utilization of yak resources, whether it is the investigation of variety resources, variety selection and matching of yak, sale, calculation of ration and dosage, etc., it is necessary to take the weight of yak as the basis [4]. Because yak is big and strong, manual measurement needs the cooperation of multiple people to complete the statistics of the whole set of body size data of a yak [5]. 
In 2003, Teng Guanghui and others began to use visual methods to measure the weight and body size of pigs [6]. In 2006, Huang Junran of Hebei Agricultural University completed the measurement of dairy cow's body size by using reference object calibration, and designed and accomplished the management system of dairy cow's image and information [7]. In 2008, Bewley used the images of lactating cows to evaluate their physical condition. When the dairy cows passed through the weighing station, the images of the cows were taken, and the USBCS and UKBCS physical condition evaluation system were used to evaluate their physical condition [8]. In 2011, Tasdemir used digital image analysis method to measure the body size data of Holstein cows, and established the weight prediction model of Holstein cows based on fuzzy rules through regression analysis of the measured data[9]. In 2013, in order to study the comparison of dairy cow contour segmentation algorithms in natural image background, T.Van Hertem and others evaluated five different target segmentation algorithms of background images in static and dynamic background, respectively. The results show that the extraction effect of dairy cow body contour in static background is obviously better than that in dynamic background[10]. In 2014, Zhang Wen of Inner Mongolia University of Science \& Technology designed and implemented a sheep physical sign monitoring system using the algorithm in OpenCV visual library[11]. In 2015, Yukako Kuzuhara et al. used ASUS Xtion Pro sensor to measure dairy cow's back posture. By measuring and recording six characteristic positions of the dairy cow, the linear regression algorithm was used to evaluate the dairy cow's physical condition. This verified that the three-dimensional camera system could be applied to cow body measurement and performance analysis[12]. In 2016, Doeschl et al. recorded the growth process of pigs by using a visual image analysis system based on a single camera, and established the relationship between pig body size parameters and time [13]. In 2021, Sun Zijie used machine vision to estimate yak weight, and adopted traditional image processing methods to obtain yak foreground images[14].

In this study, mobile devices are used to acquire images of yaks, and the parameters of yaks are accurately obtained under non-contact conditions. The research contents are as follows: 1) The acquired binocular images of yaks are trained by U-net network to get foreground images of yaks. 2) Marking the measurement points in combination with the segmented yak foreground image; finding the corresponding position in the yak depth map formed by the binocular image to obtain the position information of the yak body size measurement points; acquiring the yak body size data combining the position information of the key measurement points. 3) Finally, the yak weight was estimated. The flow chart of the measurement method in this paper is shown in Figure 1. This study can save time and labor costs, guide herders to manage yak reasonably, and provide a guarantee for the sustainable development of alpine pastoral areas.

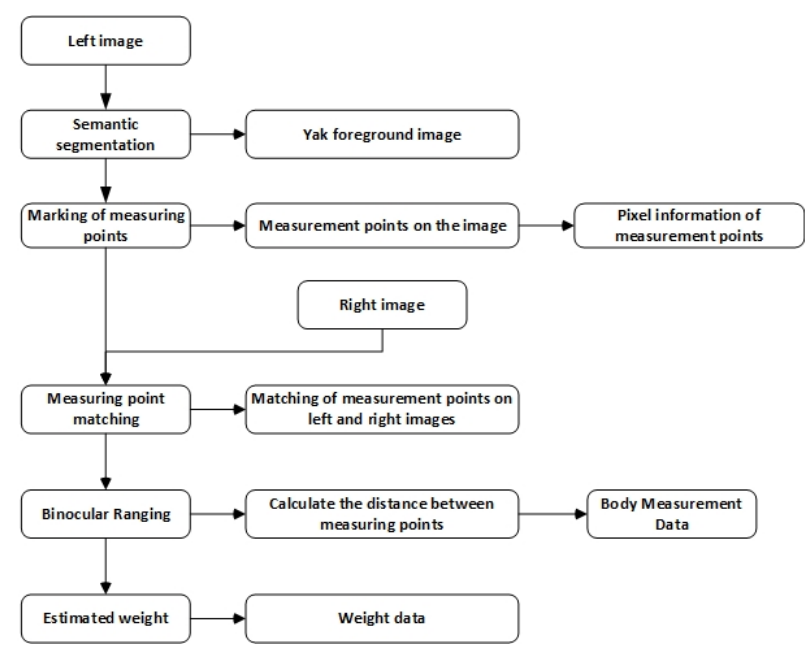

Figure 1. Flow of the Measurement Method. 


\section{Material and Method}

\subsection{Capture of Yak Image}

The yak images used in this experiment were from Qumalai County, Yushu Tibetan Autonomous Prefecture, Qinghai Province. A total of 2,263 binocular images of 200 yaks were captured, and the body size data were obtained through manual measurement. The weight data of 40 yaks were obtained through electronic weighing. The experimental image is shown in Figure 2. Part of the server platform of the test semantic segmentation is configured with Intel Xeon E5-2603 as the CPU, GeForce GTX1080Ti as the graphics card, and the binocular and weight estimation are completed on this machine with the Core i5-9400 as the CPU.

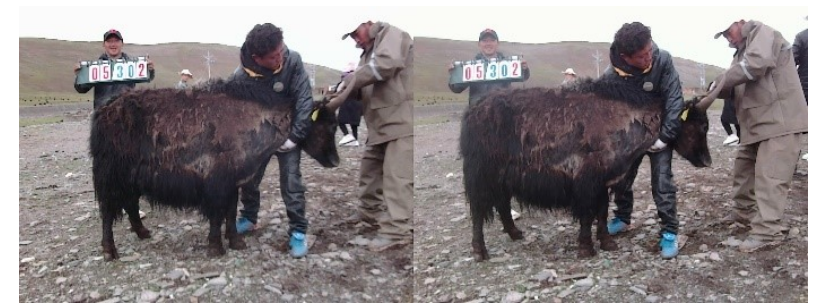

Figure 2. Experimental Image.

\subsection{Extraction of Yak Foreground Targets}

At present, many scholars have applied semantic segmentation algorithm to animal image segmentation[15]. For non-contact measurement of weight and body size of yak, firstly, the collected yak images should be separated from the background, and then the weight and body size are estimated combining the binocular vision. We use U-net algorithm to observe the overall situation firstly, roughly determine the location of the target area[16], and then consider the more detailed information for further judgment. This can make the segmentation results more accurate[17]. Therefore, the U-net algorithm is used for yak image segmentation.

The U-net algorithm was adopted for semantic segmentation of the yak's foreground and background, with two categories[18]. The image was manually segmented by Labelme, and the foreground and background of yak's image were separated to obtain a json file in coco dataset format. The image shown in Figure 3 was obtained after program processing. The data set was enhanced by increasing the sample size to 3,409 to improve the generalization ability. The obtained data set were randomly divided into the training set, the test set, and the verification set according to the ratio of 7:2:1, and the data set was input into the network of U-net[19]. The pre-training model of the VOCdevkit data set was adopted and the iteration was performed for 200 times. The random gradient descent method was used, and the learning rate was set to be 0.0001 , Batch_size to be 8 , and base_size to be 521521, then the final training model is obtained[20].

U-net, one of the earlier semantic segmentation algorithms employing full convolutional network, uses a u-shaped structure including a compression path and an expansion path. The U-net network mainly includes up-sampling part and down-sampling part to increase the receptive field and improve the most obvious characteristics of the image through maximum pooling[21,22]. In this way, the down-sampling process is completed, and the image is mapped from small resolution to large resolution by up-convolution to restore the image size and complete up-sampling. The entire network uses convolution and rectified linear function to complete the sampling of the input image and outputs a feature map. Among them, convolution is mainly used to extract local features of images, while rectified linear function is mainly used to retain relevant features and remove irrelevant features[23]. The introduction of skip-connection in U-net network can lead out shallow convolution features. In the process of up-sampling at each level, it is true that the U-net network fuses the feature map of the corresponding positions of encoders on the channel[24], thus ensuring that the finally restored images fuse more 
bottom features and features of different scales, keeping more world information in segmentation and improving segmentation accuracy.
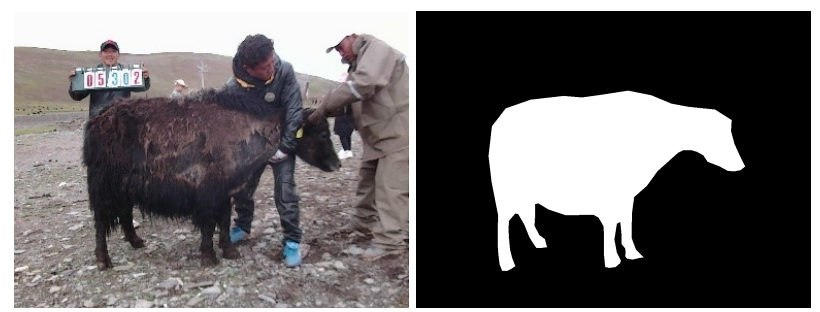

Figure 3. Original Image and Manually Segmented Image.

\subsection{Marking of Measurement Points of Yak Body Size}

After segmenting the foreground image of the yak by the U-net network, the measurement points of the body size of the yak on the foreground image are marked. Accurately marked measurement points can make people precisely find the corresponding positions of the measurement points in space on the depth image of the yak combining the binocular image, thus further exactly calculating the body size data of the yak $[25,26]$. In this experiment, the yak body height, body oblique length and chest depth were selected to calculate the yak weight, so the measurement points related to the yak body height, body oblique length and chest depth should be marked on the yak foreground image. Table 1 illustrates the positions of the measurement points in the image, and the specific positions of the measurement points are shown in Figure 4.

Table 1. Description of Corresponding Positions of Measurement Points.

\begin{tabular}{clc}
\hline Body Size & Description & Position on the Image \\
\hline Body Height & $\begin{array}{l}\text { The distance from the with- } \\
\text { ers to the ground }\end{array}$ & A1-A2 \\
Body Oblique Length & $\begin{array}{l}\text { Distance between shoulder } \\
\text { and ischial end }\end{array}$ & B1-B2 \\
Chest Depth & $\begin{array}{l}\text { Distance from back end of } \\
\text { scapula to abdomen }\end{array}$ & C1-C2 \\
\hline
\end{tabular}

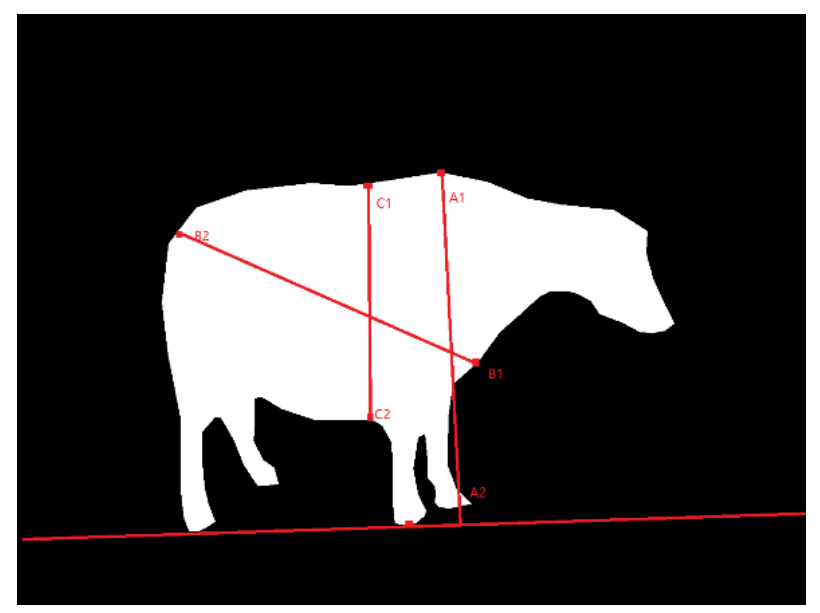

Figure 4. Original Image and Manually Segmented Image.

According to Figure 4, it is necessary to mark the measurement points of yak body size in the foreground image of yak segmented by U-net network. First, it is essential to get the yak contour from the foreground image as shown in Figure 5. Yak contour is the edge of the segmented yak foreground image, and a reflection of local color abrupt change of the image. Calculate the average value of color values in 8 directions for each 
pixel of the foreground image, and set 254 and 136 as the upper and lower thresholds. The pixels in this range are the contour of yak foreground image.

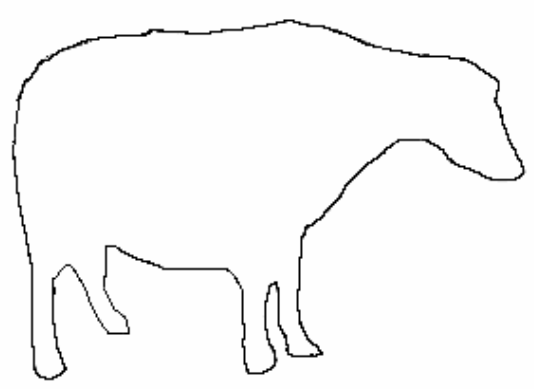

Figure 5. Contour.

After obtaining the yak contour, it is necessary to find out the six measurement points related to the yak body size as shown in Figure 4, and calculate the geometric center of the extracted yak foreground image.

\subsection{Use Binocular Vision to Measure Yak Body Size}

After the segmentation of left and right images and the marking of measurement points, we enter the binocular ranging stage. As an important branch of computer vision, binocular vision uses two cameras in different positions shoot the same scene[27]. As shown in Figure 6, the binocular camera used in this experiment can capture two images of 1280960 , and obtain the 3D coordinates of the spatial point by calculating the parallax of the spatial point in the two images[28,29]. The principle of binocular imaging is shown in Figure 7.

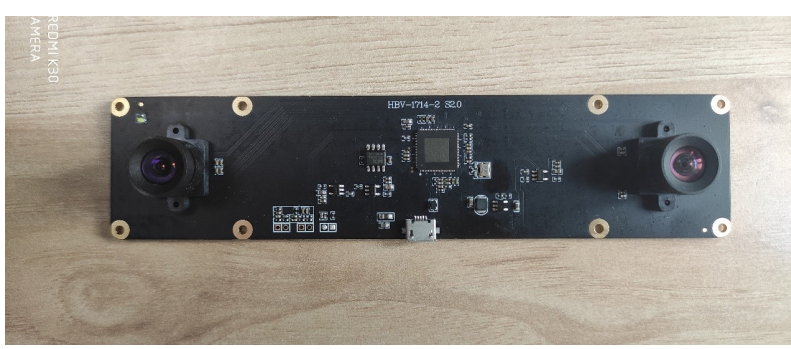

Figure 6. Binocular Camera.

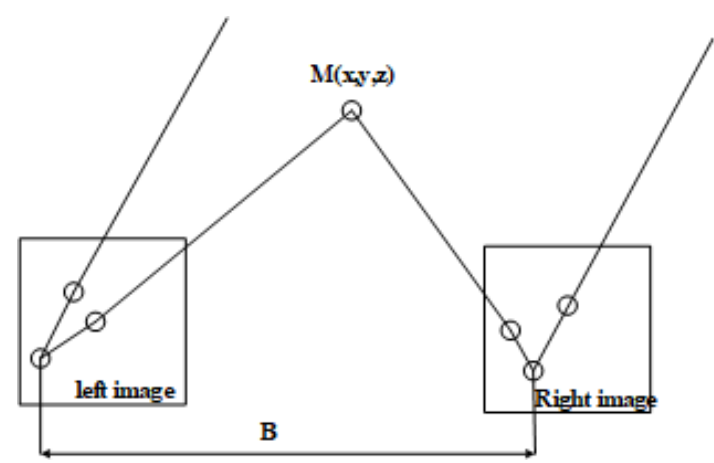

Figure 7. Principle of Binocular Imaging. 
As shown in Figure 7, B is the distance between the projection centers of the two cameras. Let's assume a measurement point $M(x, y, z)$ in space, and the image points of $\mathrm{M}$ in binocular cameras are respectively $M_{-}$left $=\left(x \_l e f t, y \_l e f t\right)$ and $M \_r i g h t=$ $\left(x \_\right.$right,y_right). Because the two cameras are in the same plane, so the coordinate $\mathrm{y}$ of the measurement point images on the left and right cameras are same. That is, $y=y_{-}$left $=y_{\text {_right }}$, and the three-dimensional coordinates of $\mathrm{M}$ can be obtained from the triangular geometric relationship as follows: $x=\left(B x \_l e f t\right) / d, y=B y / d, z=B f / d$, where $\mathrm{f}$ is the focal length of the camera $d=x \_$left $-x$ _right $[30,31]$. Therefore, any point on the image of left camera can determine the 3D coordinates of the point as long as the corresponding matching point can be found on the image of right camera[32].

Before binocular ranging, it is necessary to calibrate the camera to obtain intrinsic and extrinsic parameters[33]. We select Zhang Youzheng chessboard calibration method[34], and obtain the focal length $\mathrm{f}$, baseline distance B of the binocular camera as well as the translation vector $T$ and rotational vector $R$ between two cameras through calibration. Table 2 shows the data obtained by binocular calibration.

Table 2. Calibration Data.

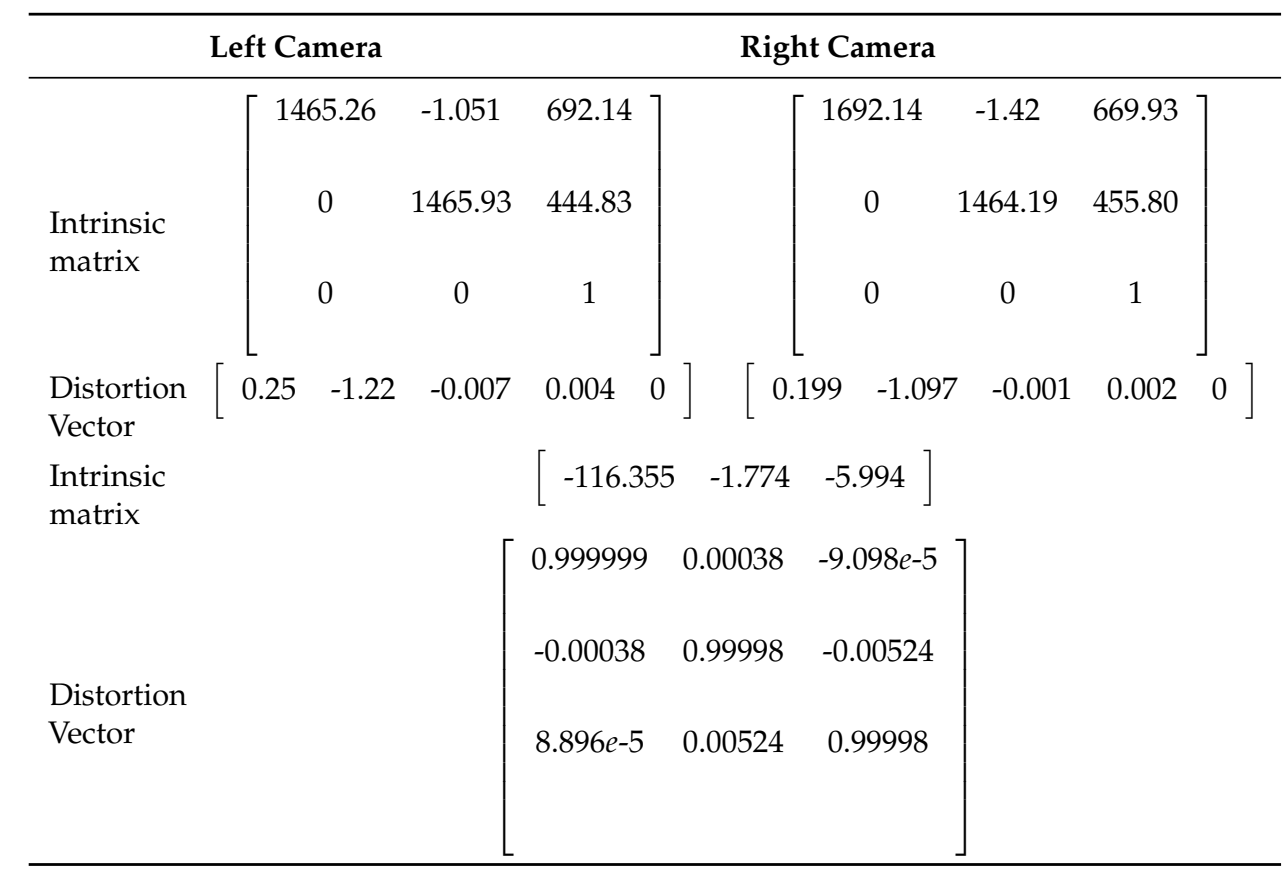

According to the principle of three-dimensional coordinates of a point in the binocular measured image and the data obtained by camera calibration, combined with the measurement points found in the foreground image, the corresponding positions of the measurement points can be found in the depth image, thus obtaining the threedimensional coordinates and yak body size parameters.

\subsection{Yak Weight Estimation}

There is a high correlation between the weight and body size of yak[35]. In this study, the body height, body oblique length and chest depth of yak were selected for modeling by using method such as linear regression[36] and support vector regression[37]. Six yak weight prediction models were established based on the old and new data. The correlation between body size and weight was analyzed by using 3581 data. The results are shown in Table 3. It can be seen that the body size data has a high correlation with weight. In the data set, $\mathrm{W}, \mathrm{tg}, \mathrm{tx}, \mathrm{xw}$ indicate the weight, the body height, the body oblique length, and the chest circumference respectively. 
Table 3. Correlation Coefficient Between Body Size and Weight of Yak.

\begin{tabular}{ccccc}
\hline & $\mathbf{W}$ & tg & tx & xw \\
\hline $\mathrm{W}$ & 1.000000 & 0.749930 & 0.653193 & 0.693756 \\
$\mathrm{tg}$ & 0.749930 & 1.000000 & 0.636013 & 0.649566 \\
$\mathrm{tx}$ & 0.653193 & 0.636013 & 1.000000 & 0.543669 \\
$\mathrm{xW}$ & 0.693756 & 0.649566 & 0.543669 & 1.000000 \\
\hline
\end{tabular}

In the linear regression algorithm model, each data has $\mathrm{n}$ features, and each feature corresponds to its own weight value, and the product with the weight plus an offset value is the linear regression model[38]. The formula is as follows:

$$
y=w_{1} * x_{1}+w_{2} * x_{2}+\ldots+w_{n} * x_{n}+b
$$

If $w_{0}=b, x_{0}=1$, then you can get:

$$
y=w_{0} * x_{0}+w_{1} * x_{1}+w_{2} * x_{2}+\ldots+w_{n} * x_{n}
$$

Now there are $\mathrm{m}$ samples to get the matrix represented as:

$$
X=\left[\begin{array}{ccccc}
1 & x_{1}^{1} & x_{1}^{2} & \ldots & x_{1}^{n} \\
1 & x_{2}^{1} & x_{2}^{2} & \ldots & x_{2}^{n} \\
\ldots & \ldots & \ldots & \ldots & \ldots \\
1 & x_{m}^{1} & x_{m}^{2} & \ldots & x_{m}^{n}
\end{array}\right] Y=\left[\begin{array}{c}
y_{1} \\
y_{2} \\
\ldots \\
y_{m}
\end{array}\right]
$$

Among them, the weight $\mathrm{w}$ is represented as:

$$
w=\left[\begin{array}{lllll}
w_{0} & w_{1} & w_{2} & \ldots & w_{n}
\end{array}\right]
$$

The model function of support vector regression model is also a linear function, but it is different from linear regression model in the principle of calculating loss as well as the objective function and optimization algorithm. A "marginal zone" is created on both sides of the linear function of support vector regression, and no loss is calculated for all samples falling into the marginal zone[39]. Only those outside the marginal zone are included in the loss function. Then, the model is optimized by minimizing the width and total loss of the marginal zone.

\section{Experimental Results and Analysis}

\subsection{Evaluation of Foreground Extraction Results}

The evaluation indexes of semantic segmentation mainly include three indexes: execution time, memory usage and accuracy. Considering this experiment, four kinds of semantic segmentation accuracy evaluation indexes are used to evaluate the segmentation model[40,41]. They are PA (pixel accuracy), CPA (category pixel accuracy), MPA (mean pixel accuracy) and MIoU (mean intersection over union).

Assuming that there must be $k+1$ categories (including $\mathrm{k}$ target categories and 1 background category), the pixels with $i$ as $p_{i} i$ identification category are predicted as the total number of $i$; the pixels with $i$ as $p_{i j}$ identification category are predicted as the total number of $j$; and the pixels with $j$ as $p_{j i}$ identification category are predicted as the total number of $i$. 
PA is the pixel accuracy, that is the ratio of the number of correctly classified pixels to the number of all pixels is represented as:

$$
P A=\frac{\sum_{i=0}^{k} p_{i i}}{\sum_{i=0}^{k} \sum_{j=0}^{k} p_{i j}}
$$

CPA is the category pixel accuracy, that is the accuracy of pixels that really belong to category $i$ in category $i$ prediction is represented as:

$$
C P A_{i}=\frac{\sum_{i=0}^{k} p_{i i}}{\sum_{i=0}^{k} p_{i i}+\sum_{j=0}^{k} p_{i j}}
$$

MPA is the mean pixel accuracy, that is the average value of the ratio between the number of correctly classified pixels in each category and the number of all pixels in that category is represented as:

$$
M P A=\frac{1}{k} \sum_{i=1}^{k} \frac{p_{i i}}{\sum_{i=0}^{k} p_{i j}}
$$

MIoU is the mean intersection over union, that is the average value of IoU of each category (the ratio of intersection and union of the predicted results and true values of a certain category of the model) is represented as:

$$
\text { MIoU }=\frac{1}{k+1} \sum_{i=1}^{k} \frac{p_{i i}}{\sum_{i=0}^{k} p_{i j}+\sum_{i=0}^{k} p_{j i}-p_{i i}}
$$

In this study, the training was repeated many times, and the results of the model prediction accuracy obtained are shown in Table 4.

Table 4. Evaluation of Model Prediction Accuracy.

\begin{tabular}{cccccc}
\hline No. & PA & $\mathbf{C P A}_{0}$ & $\mathbf{C P A}_{1}$ & MPA & MIoU \\
\hline 1 & 0.9831 & 0.9863 & 0.9714 & 0.9789 & 0.9506 \\
2 & 0.9839 & 0.9868 & 0.9728 & 0.9798 & 0.9535 \\
3 & 0.9834 & 0.9884 & 0.9658 & 0.9771 & 0.9519 \\
4 & 0.9831 & 0.9849 & 0.9761 & 0.9805 & 0.9510 \\
5 & 0.9836 & 0.9857 & 0.9753 & 0.9805 & 0.9520 \\
6 & 0.9833 & 0.9856 & 0.9746 & 0.9801 & 0.9516 \\
7 & 0.9825 & 0.9854 & 0.9722 & 0.9788 & 0.9490 \\
8 & 0.9842 & 0.9871 & 0.9731 & 0.9801 & 0.9537 \\
9 & 0.9831 & 0.9854 & 0.9753 & 0.9803 & 0.9514 \\
10 & 0.9833 & 0.9873 & 0.9696 & 0.9784 & 0.9515 \\
Mean Value & 0.9833 & 0.9862 & 0.9726 & 0.9794 & 0.9516 \\
\hline
\end{tabular}

From Table 6, it can be seen that after 10 times of repeated training, the average PA of the model is 0.9833 . For CPA, the background accuracy and the foreground accuracy is 0.9862 and 0.9722 respectively. The MPA is 0.9794 , and the MIoU is 0.9516 . This shows that the model has good stability and generalization ability.

By comprehensively comparing the results of 10 times training, the accuracy of the eighth training is higher. Therefore, the U-net network structure of the eighth training is stored for subsequent direct call. We can use the trained model for yak image segmentation. The results are shown in Figure 8. According to the segmentation results of yak image, it can be seen that the segmentation of yak foreground image based on U-net network built in this experiment have better results, and it also has better segmentation performance on complex background images. 
(a)

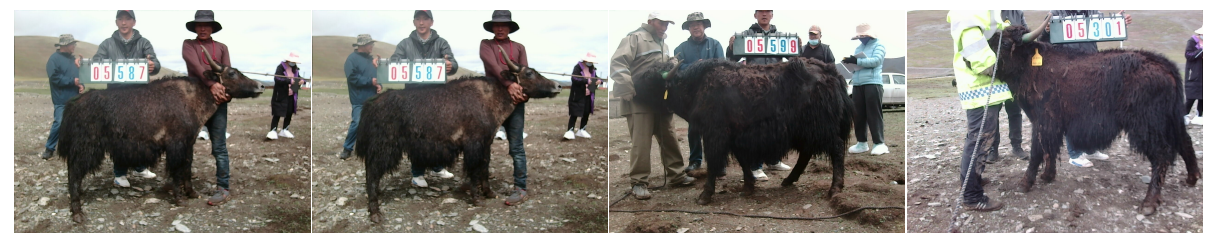

(b)

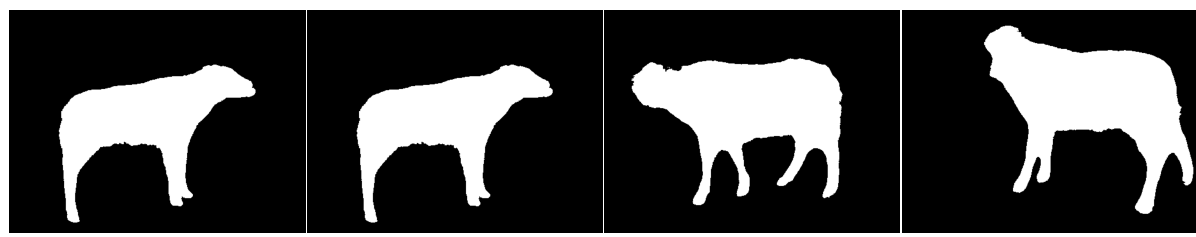

Figure 8. Yak Image Segmentation Result. (a) Original Image. (b) Segmented Image.

\subsection{Evaluation of Measurement Results of Yak Body Size}

Popriate binocular images need to be selected for the experiment. We use U-net network to get the left and right yak foreground images, and find the measurement points related to body size on the foreground images, as shown in Figure 9. It is the marked measurement points related to body size. Then we get the pixel information related to the measurement points, and obtain the depth map in combination with the binocular images and find the positions corresponding to the measurement points in the depth map. Furthermore, we acquire the yak body size data, including body height, body oblique length and chest depth. Table 5 is the result statistics of body size measurement data, including data of 90 yaks.

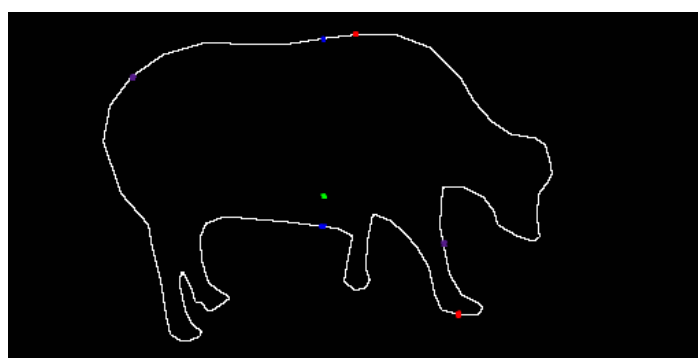

Figure 9. Measurement Point.

Table 5. Result Statistics of Body Size Measurement.

\begin{tabular}{cccc}
\hline & Max error/cm & Min Error/cm & Ave Error/cm \\
\hline Body Height & 4.699 & 1.121 & 2.594 \\
Body Oblique Length & 5.329 & 1.147 & 2.666 \\
Chest Depth & 11.996 & 1.486 & 5.94 \\
\hline
\end{tabular}

The data of 10 yaks were randomly selected to show the results, as shown in Table 6 
Table 6. Part of Yak Data.

\begin{tabular}{ccccccccc}
\hline \multicolumn{3}{c}{ Body Height } & \multicolumn{3}{c}{ Body Oblique Length } & \multicolumn{3}{c}{ Chest Depth } \\
\hline \multirow{2}{*}{ Manual } & Binocular & Error & Manual & Binocular & Error & Manual & Binocular & Error \\
\hline 104 & 106.045 & $1.97 \%$ & 109 & 106.92 & $1.91 \%$ & 40 & 42.91 & $7.80 \%$ \\
104 & 108.699 & $4.52 \%$ & 108 & 110.055 & $1.90 \%$ & 40 & 42.76 & $6.57 \%$ \\
104 & 107.252 & $3.13 \%$ & 110 & 108.406 & $1.45 \%$ & 39 & 41.77 & $7.50 \%$ \\
106 & 109.249 & $3.07 \%$ & 114 & 119.329 & $4.68 \%$ & 43 & 41.56 & $2.62 \%$ \\
105 & 103.872 & $1.07 \%$ & 109 & 106.286 & $2.49 \%$ & 42 & 46.14 & $8.94 \%$ \\
104 & 106.311 & $2.22 \%$ & 110 & 113.123 & $2.84 \%$ & 43 & 46.5 & $8.95 \%$ \\
105 & 103.676 & $1.26 \%$ & 115 & 112.593 & $2.09 \%$ & 46 & 47.62 & $3.83 \%$ \\
106 & 109.622 & $3.42 \%$ & 113 & 115.007 & $1.78 \%$ & 44 & 45.91 & $4.45 \%$ \\
105 & 108.906 & $3.72 \%$ & 112 & 116.446 & $3.97 \%$ & 45 & 43.87 & $2.31 \%$ \\
104 & 108.501 & $4.33 \%$ & 109 & 104.463 & $4.16 \%$ & 45 & 45.49 & $1.31 \%$ \\
\hline
\end{tabular}

Yak body size measurement based on binocular vision. It combines the foreground image obtained by U-net network, and finds the relevant measurement points on the foreground image, then matches the features on the binocular image and seeks out the measurement points to obtain yak body size data. According to the test results, the measurement results of this method are not much different from the manually measured yak body size data, but the overall cost will be much less than that of manual measurement. It can save the time and labor cost in measuring yak body size data.

\subsection{Evaluation of Yak Weight Estimation Results}

3580 yaks body size and weight data are selected to fit the model which predicting weight with yak body size. Among them, the data of 200 yaks are new. Linear regression algorithm and support vector regression algorithm are adopted to get the yak weight prediction model. The overall performance of the four yak weight prediction models on the test data are as shown in Figure 10.

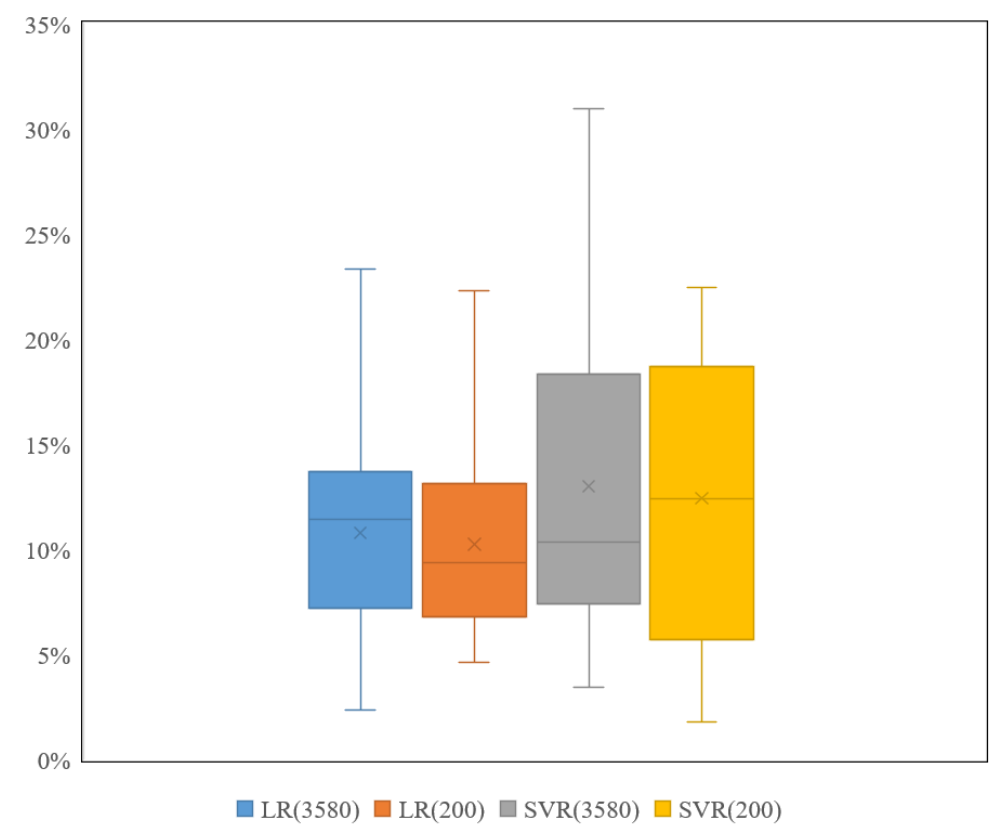

Figure 10. Yak weight estimates the overall performance of the model.

The yak body size data obtained by binocular measurement is used to estimate the yak weight. For example, Table 7 shows the average error and fitting equation of the four models on 90 groups of test data. 
Table 7. Result Statistics of Body Size Measurement.

\begin{tabular}{ccc}
\hline Method & Equation & AVG /\% \\
\hline LR (3580) & $W=3.7775 * B H+1.0258 * B O L+0.8317 * C D-502.0926$ & 10.78 \\
SVR (3580) & & 13.01 \\
LR (200) & $W=3.8724 * B H+2.0787 * B O L+0.1364 * C D-478.207$ & 10.26 \\
SVR (200) & & 12.44 \\
\hline
\end{tabular}

\section{Conclusion and discussion}

In this paper, the images taken by binocular cameras are used as experimental materials to estimate yak's body size and weight under non-contact conditions. The experimental results show: (1) The segmentation accuracy of yak image foreground extraction algorithm based on U-net network is over 97\%. (2) Based on binocular vision, the average measurement error of yak body height and body oblique length is $2.6 \%$, and the average measurement error of yak chest depth is $7 \%$. (3) We estimate the yak weight according to yak body size data based on semantic segmentation and obtained by binocular vision, the average errors of the four prediction models are $10.78 \%$, $13.01 \%, 10.26 \%$ and $12.44 \%$, respectively. Based on the above results, the non-contact measurement method of yak body size and weight proposed in this experiment have high accuracy, and can effectively solve the time and labor consuming problem in the process of yak body size and weight measurement under the condition of ensuring the accuracy of binocular images.

Author Contributions: Conceptualization, W.W. and Y.Z.; methodology, W.W.; software, W.W.; vali-dation, S.Z., G.L. and W.Z.; formal analysis, W.W.; investigation, Y.B.,Q.B.,and X.L.; resources, J.H.,Z.C.,C.M.and D.L.; data curation,W.W.; writing-original draft preparation, W.W.; writing-review and editing, W.W.; visualization,W.W.; supervision, R.S.; funding acquisition, Y.Z. All authors have read and agreed to the published version of the manuscript.

Funding: This work is supported by Science and Technology Project in Qinghai Province (No: 2020-QY-218); Supported by China Agriculture Research System of MOF and MARA(CARS-37); And received the support of the "High-end Innovative Talents Thousand Talents Program" in Qinghai Province.

Institutional Review Board Statement: Not applicable.

Informed Consent Statement: Not applicable.

Data Availability Statement: Limited data available on request due to the large size of the data.

Conflicts of Interest: The authors declare no conflict of interest.

\section{Abbreviations}

The following abbreviations are used in this manuscript:

$\begin{array}{ll}\text { LR } & \text { Linear Regression } \\ \text { SVR } & \text { Support Vector Machine Regression } \\ \text { W } & \text { Weight } \\ \text { BH } & \text { Body Height } \\ \text { BOL } & \text { Body Oblique Length } \\ \text { CD } & \text { Chest Depth } \\ \text { AVG } & \text { Average Value }\end{array}$

\section{References}

1. A. Cy, B. Gt, A. Qf, A. Sw, A. Zw, A. Sc, and A. Fh, “Behavioral patterns of yaks ( bos grunniens ) grazing on alpine shrub meadows of the qinghai-tibetan plateau," Applied Animal Behaviour Science, vol. 234, 2020.

2. D. U. Zi-Yin, Y. J. Cai, X. D. Wang, and B. Zhang, "Research progress on yak grazing behavior and its influence on the soil properties of alpine grassland," Acta Prataculturae Sinica, 2019. 
3. Qi, Yan, Luming, Ding, Haiyan, Wei, Xianju, Wang, and Cuixia, “Body weight estimation of yaks using body measurements from image analysis," Measurement, 2019.

4. C. Huang, F. Ge, W. Ren, Y. Zhang, and C. Liang, "Copy number variation of the hpgds gene in the ashidan yak and its associations with growth traits," Gene, vol. 772, no. 1, p. 145382, 2020.

5. Y. Qiao, H. Kong, C. Clark, S. Lomax, and S. Sukkarieh, “Intelligent perception for cattle monitoring: A review for cattle identification, body condition score evaluation, and weight estimation," Computers and Electronics in Agriculture, vol. $185,2021$.

6. Li. C, G. Teng , C. Zhao, "Using computer vision technology to realize non-destructive monitoring of greenhouse plant growth," Transactions of the Chinese Society of Agricultural Engineering, vol. 03,pp.140-143, 2003.

7. H. J, "Research on Web-based Cow Image Recognition and Image Information Management System," Hebei Agricultural University,2006.

8. J. M. Bewley, A. M. Peacock, O. Lewis, R. E. Boyce, D. J. Roberts, M. P. Coffey, S. J. Kenyon, and M. M. Schutz, “Potential for estimation of body condition scores in dairy cattle from digital images," Journal of Dairy Science, vol. 91, no. 9, pp. 3439-3453, 2008.

9. S. Tasdemir, A. Ürkmez, and S. Inal, "A fuzzy rule-based system for predicting the live weight of holstein cows whose body dimensions were determined by image analysis," Turkish Journal of Electrical Engineering E Computer Sciences, vol. 19, no. 4, pp. 689-703, 2011.

10. T. V. Hertem, V. Alchanatis, A. Antler, E. Maltz, I. Halachmi, A. Schlageter-Tello, C. Lokhorst, S. Viazzi, C. Romanini, and A. Pluk, "Comparison of segmentation algorithms for cow contour extraction from natural barn background in side view images," Computers \& Electronics in Agriculture, vol. 91, pp. 65-74, 2013.

11. W. Zhang, "Research on livestock physical sign measurement system based on machine vision," Inner Mongolia University of Science and Technology, 2014.

12. Y. Kuzuhara, K. Kawamura, R. Yoshitoshi, T. Tamaki, S. Sugai, M. Ikegami, Y. Kurokawa, T. Obitsu, M. Okita, and T. Sugino, “A preliminarily study for predicting body weight and milk properties in lactating holstein cows using a three-dimensional camera system," Computers and Electronics in Agriculture, vol. 111, pp. 186-193, 2015.

13. A. B. Doeschl-Wilson, C. T. Whittemore, P. W. Knap, and C. P. Schofield, "Using visual image analysis to describe pig growth in terms of size and shape," Animal Science An International Journal of Fundamental E Applied Research, vol. 79, no. 03, pp. 415-427, 2016.

14. Zhang. Y A, Sun. Z J, Zhang. C. Whittemore, P. W. Knap, and C. P. Schofield, “Body weight estimation of yak based on cloud edge computing," J Wireless Com Network 2021 .vol. 6,2021.

15. S. Zhao, G. Hao, Y. Zhang, and S. Wang, "A real-time semantic segmentation method of sheep carcass images based on icnet," Journal of Robotics, vol. 2021, no. 2, pp. 1-12, 2021.

16. O. Ronneberger, P. Fischer, and T. Brox, “U-net: Convolutional networks for biomedical image segmentation," in International Conference on Medical Image Computing and Computer-Assisted Intervention, 2015.

17. G. Martinez-Soltero, A. Y. Alanis, N. Arana-Daniel, and C. Lopez-Franco, "Semantic segmentation for aerial mapping," 2020.

18. D. Xu, X. Zhou, X. Niu, and J. Wang, "Automatic segmentation of low-grade glioma in mri image based on unet++ model," Journal of Physics: Conference Series, vol. 1693, no. 1, p. 012135 (7pp), 2020.

19. I. C. Saidu and L. Csató, "Active learning with bayesian unet for efficient semantic image segmentation," Journal of Imaging, vol. 7, no. 2, p. 37, 2021.

20. H. Jiang and Y. E. Xining, “An improved skin disease image segmentation algorithm based on i-unet network," Modern Electronics Technique, 2019.

21. Y. A. Ayalew, A. Kinde, and M. A. Mohammed, "Modified u-net for liver cancer segmentation from computed tomography images with a new class balancing method," BMC Biomedical Engineering, vol. 3, no. 1, 2021.

22. S. T. Tran, C. H. Cheng, T. T. Nguyen, M. H. Le, and D. G. Liu, “Tmd-unet: Triple-unet with multi-scale input features and dense skip connection for medical image segmentation," Healthcare, vol. 9, no. 1, p. 54, 2021.

23. Y. F. Zhang, J. Zheng, L. Li, N. Liu, and X. He, "Rethinking feature aggregation for deep rgb-d salient object detection," Neurocomputing, vol. 423, no. 4, pp. 463-473, 2021.

24. A. E. Maxwell, M. S. Bester, L. A. Guillen, C. A. Ramezan, and J. L. Pyron, “Semantic segmentation deep learning for extracting surface mine extents from historic topographic maps," Remote Sensing, vol. 12, no. 24, p. 4145, 2020.

25. P. Azad, Visual Perception for Manipulation and Imitation in Humanoid Robots. Visual Perception for Manipulation and Imitation in Humanoid Robots, 2009.

26. L. Li, B. Li, Z. Wu, D. Gao, and X. Mu, "Automatic placement of annotation in area feature by map spatial geometry information measurement," Proceedings of SPIE - The International Society for Optical Engineering, vol. 6751, 2007.

27. L. Zhang, C. Li, Y. Fan, X. Zhang, and J. Zhao, "Physician-friendly tool center point calibration method for robot-assisted puncture surgery," Sensors, vol. 21, no. 2, p. 366, 2021.

28. Z. C. Qiu and Z. Q. Huang, "A shape reconstruction and visualization method for a flexible hinged plate using binocular vision," Mechanical Systems and Signal Processing, vol. 158.

29. D. Ruan, W. Zhang, and D. Qian, "Feature-based autonomous target recognition and grasping of industrial robots," Personal and Ubiquitous Computing, no. 1, pp. 1-13, 2021.

30. F. Jin and Y. H. Fan, "Digital image stabilization with motion estimation based on binocular ranging and sensor," in Chinese Control Conference, 2014. 
31. T. Shen, W. Liu, and J. Wang, "Distance measurement system based on binocular stereo vision," Electronic Measurement Technology, 2015.

32. C. Shi, J. Zhang, and G. Teng, "Mobile measuring system based on labview for pig body components estimation in a large-scale farm," Computers and Electronics in Agriculture, vol. 156, pp. 399-405, 2019.

33. Dingfei, Jin, Yue, and Yang, "Using distortion correction to improve the precision of camera calibration," Optical Review, vol. 26, no. 2, p. 269-277, 2019.

34. A. Zisserman, “Book review : Epipolar geometry in stereo, motion and object recognition—a unified approach by gang xu and zhengyou zhang published by kluwer academic publishers group; 1996; 313 pages; us $\$ 160$," International Journal of Robotics Research, vol. 17, no. 8, pp. 903-904, 1998.

35. A. J. da Silva Cardoso, C. A. L. D. Oliveira, E. C. Campos, R. P. Ribeiro, and F. F. E. Silva, "Estimation of genetic parameters for body areas in nile tilapia measured by digital image analysis," Journal of Animal Breeding and Genetics, 2021.

36. M. Lira, E. Silva, J. M. B. Alves, and G. Veras, "Estimate of wind resources in the coast of ceará using the linear regression theory," Renewable E Sustainable Energy Reviews, vol. 39, no. nov., pp. 509-529, 2014.

37. T. K. Lin, T. H. Yang, P. H. Wang, R. C. Zeng, and K. C. Chang, "Prediction of smooth hysteretic model parameters using support vector regression," Multiscal

38. L. I. Dahu, "Predicting short-term traffic flow in urban based on multivariate linear regression model," ournal of Intelligent and Fuzzy Systems.vol 39, pp. 1-11, 2020.

39. P. Anand, R. Rastogi, S. Chandra, A class of new support vector regression models, Applied Soft Computing (2020) 106446.

40. M. Shahzad, A. I. Umar, M. A. Khan, S. H. Shirazi, Z. Khan, W. Yousaf, Robust method for semantic segmentation of whole-slide blood cell microscopic image, Computational and Mathematical Methods in Medicine 2020 (2020) 1-13.

41. F. Lateef, Y. Ruichek, Survey on semantic segmentation using deep learning techniques, Neurocomputing 338 (2019) 321-348 